
internationales

vol. 21 - n³ | 2005

Familles, destins personnels et appartenances

collectives en migration

\title{
Engagement associatif et individuation de femmes migrantes
}

Blandine Veith

\section{CpenEdition}

Journals

Édition électronique

URL : https://journals.openedition.org/remi/2512

DOI : $10.4000 /$ remi. 2512

ISSN : $1777-5418$

Éditeur

Université de Poitiers

Édition imprimée

Date de publication : 1 décembre 2005

Pagination : 53-67

ISBN : 2-911627-45-8

ISSN : 0765-0752

Référence électronique

Blandine Veith, «Engagement associatif et individuation de femmes migrantes », Revue européenne des migrations internationales [En ligne], vol. 21 - n³ | 2005, mis en ligne le 01 décembre 2008, consulté le 16 avril 2022. URL : http://journals.openedition.org/remi/2512 ; DOI : https://doi.org/ $10.4000 /$ remi. 2512

Ce document a été généré automatiquement le 16 avril 2022

(c) Université de Poitiers 


\title{
Engagement associatif et individuation de femmes migrantes
}

\author{
Blandine Veith
}

1 Cet article s'attache à l'étude de la construction de l'individu moderne dans une perspective de genre qui prend en compte les effets de migrations internationales et d'un engagement associatif. Il s'agit de comprendre en quoi des mobilités, de pays du Sud vers la France, sont des freins ou des incitations au processus d'individuation que des femmes ont engagé au cours de leur vie; en quoi et comment une implication durable en France dans des associations multiculturelles peut être pour ces femmes une voie spécifique de l'individuation. Une individuation qui est à la fois une aspiration à maîtriser le cours de sa vie et une injonction à être soi.

2 Comment celles qui cherchent à se dégager des rapports de dépendance pour avoir plus d'emprise sur leur vie, pour construire des projets et des stratégies, concilient-elles ces souhaits avec le maintien de liens forts avec leurs communautés et avec la valorisation de leurs cultures d'origine? Elles arrivent à l'âge adulte dans un pays où le système dominant de normes et de valeurs accorde une place centrale à l'individu (Dumont, 1983), où la propriété sociale est censée garantir à la plupart la propriété de soi (Castel, 2001). Le changement de contexte de vie peut induire, renforcer ou au contraire contrecarrer une individuation déjà en cours. Cependant la perspective n'est pas d'opposer tradition et modernité (Dumont, 1991 : p. 30-32) en associant la première aux sociétés d'origine et la seconde à la société d'arrivée. Il s'agit au contraire de montrer comment tradition et modernité peuvent être en tension et se combiner dans des contextes divers et précis, ceux qui sont évoqués par les personnes qui racontent leur vie avant et après la migration. En effet, d'un côté ces femmes viennent de sociétés post-coloniales en contact avec les valeurs individualistes. De l'autre, elles suivent ou rejoignent en France un conjoint dans le cadre d'un regroupement familial : elles sont assignées à des fonctions d'épouse et de mère et sont perçues comme telles tant par les familles que par les autorités administratives. Il s'agit d'interroger aussi les supports de leur individuation. La place faite en France aux familles migrantes venues de pays du Sud - la ségrégation spatiale, la précarisation sociale qui les touchent plus 
particulièrement, les discriminations dans l'accès au travail, au logement, à la santé peuvent les enfermer dans des cadres spatio-temporels qui limitent la projection dans l'espace et dans l'avenir (Appay et Léomant, 1999). L'accès restreint à des droits civils, politiques et sociaux fondamentaux (Marshall, 1950) d'une part, l'isolement et la coupure avec les réseaux de solidarité féminine d'autre part peuvent favoriser, dans un premier temps, une plus grande soumission aux hiérarchies sociales et sexuées héritées. C'est pourquoi nous nous intéresserons non seulement aux supports de l'individuation (Castel, 2001) mais aussi aux ressorts de l'action : aux dispositions issues de la pluralité des socialisations et aux contextes favorisant leur expression (Lahire, 1998). L'objectif est de comprendre comment des femmes, exclues du marché du travail ou reléguées dans des emplois précaires et peu qualifiés, tentent d'accéder en France à l'individuation à travers l'engagement associatif. En quoi et comment l'implication dans des associations multiculturelles peut-elle être pour ces femmes une façon spécifique de s'individuer? Quels sont les effets de cet engagement sur leur devenir?

3 À partir de ces interrogations, je me propose donc d'analyser les parcours biographiques d'une catégorie spécifique de primo-migrantes: celles qui à l'issu d'un engagement militant durable, ont créé leur emploi au sein d'associations ou ont monté, avec leur appui, des collectifs de travail, tels des restaurants, des ateliers de couture ou de coiffure. Ces collectifs ont été fondés par des femmes de diverses origines géographiques, religieuses, sociales et ont été mis en exergue par les médias. Situés dans des banlieues souvent stigmatisées, ils ont bénéficié du soutien d'associations féminines et multiculturelles de quartier ainsi que de subventions publiques, notamment celles du Fasild et de la politique de la Ville. Toutefois, comment ces mères de familles nombreuses peuvent-elles se lancer dans de tels projets alors qu'elles sont parfois illettrées, pas ou peu qualifiées? Quelles ressources liées à leur histoire personnelle et sociale ont-elles alors mobilisées? La plupart n'avaient jamais eu en France un travail rémunéré; les autres étaient au chômage et n'avaient eu que des parcours professionnels caractérisés par une succession discontinue d'emplois précaires, souvent à temps partiel, aux horaires flexibles et décalés: des parcours de « combattants bricoleurs" pour reprendre l'expression de l'une d'entre elles. Si créer son emploi apparait alors comme la seule façon d'en obtenir un ou de maîtriser ses conditions de travail, jusqu'à quel point ces micro-entreprises échappent-elles à la précarisation sociale? Le propos ici n'est pas de traiter de cette question abordée dans des travaux antérieurs (Veith, 1998). Notons toutefois que celles qui ont pu se lancer de façon durable dans cette opération longue et incertaine font généralement partie de familles aux revenus stables même s'ils restent modestes. En effet, ces femmes acceptent souvent, au nom de la survie de leur commerce ou artisanat, des rémunérations trop faibles pour en vivre. Elles sont aussi conduites à s'imposer des horaires flexibles et extensibles, avec des conditions de travail difficiles où elles usent leur santé. Elles n'échappent donc pas aux effets de la précarisation sociale (Appay et Thébaud-Mony, 1997) et la viabilité de ces micro-entreprises et associations reste très incertaine. Quel est alors l'impact de ces projets associatifs sur l'accès à l'individuation de ces femmes dans un tel contexte socio-économique?

4 Pour répondre à ces interrogations, je mobilise le matériau d'une recherche qualitative initiée dans le cadre d'un travail collectif consacré aux conséquences de la précarisation sociale sur la vie de famille. Des récits de vie et des entretiens biographiques ont été recueillis dans quatre projets situés dans des banlieues populaires de la région parisienne. Ils ont été croisés au niveau des familles, des 
collectifs de travail ou des associations et ils ont été complétés par une observation participante dans un restaurant, puis dans l'association qui l'a soutenu et par la réalisation de monographies d'associations. J'ai en outre suivi depuis 1997 l'évolution de familles impliquées dans ces projets. Les récits de vie de primo-migrantes venues de différents continents ont été dans un premier temps comparés, afin de repérer les récurrences et faire émerger des hypothèses. Ces récits ont été ensuite analysés au niveau de leur cohérence interne en étant replacés dans leur contexte culturel, familial, professionnel et associatif.

5 Ce va-et-vient entre les deux niveaux d'analyse a permis de construire un objet de recherche centré sur l'individuation de ces femmes et d'affiner les hypothèses. En montant, au sein d'associations multiculturelles, des projets professionnels, ces femmes deviennent plus indépendantes financièrement et développent des capacités à prendre la parole en public, à se déplacer, à construire des stratégies, ce qui les conduit à renégocier progressivement les rapports de genre au sein des familles. Elles aspirent à continuer en France une individuation qu'elles avaient, presque toutes, amorcée dans leurs pays d'origine, chacune à sa façon, avec des degrés divers de complexité et dans des contextes spécifiques. Elles racontent aujourd'hui qu'elles avaient commencé à s'y affirmer en revendiquant le droit à la scolarité, au choix du conjoint, à l'indépendance financière, parfois à l'expression et à la participation politique. Elles présentent leurs trajectoires en développant le même argumentaire et en mobilisant les mêmes critères. Il s'agit d'aborder ici cet aspect des parcours de vie pour mieux saisir les difficultés et les dynamiques actuelles.

6 S'intéresser aux caractéristiques socio-économiques et culturelles de ces femmes ne suffirait pas pour comprendre comment elles tentent de résister aux rapports de domination qu'elles subissent en tant que migrantes venues de pays du Sud qui furent souvent d'anciennes colonies, en tant que femme et en tant que membre des classes populaires reléguées dans des quartiers stigmatisés. Certes ces femmes sont des urbaines: elles ont, pour la plupart, vécu leur enfance dans de grandes villes. Certes elles sont issues de familles qui ne sont ni les plus pauvres ni les plus riches: des familles d'artisans et commerçants, de petits et moyens entrepreneurs, de cadres de la Fonction publique, des familles qui sont à l'abri du besoin, qui peuvent parfois avoir une position élevée dans les hiérarchies locales. Certaines femmes ont été scolarisées, parfois jusqu'au baccalauréat, et ont eu une activité lucrative. D'autres ont appris à parler plusieurs langues ou dialectes. Elles ne sont donc pas démunies de ressources. Cependant, en France, elles ne sont pas perçues comme telles puisqu'elles occupent le bas de l'échelle sociale. À leur arrivée, elles ont subi un déclassement. Même si les capitaux sociaux, relationnels et culturels hérités ne doivent pas être occultés, leur seule analyse ne permet cependant pas de comprendre les ressorts de l'action de ces femmes.

7 Celles-ci ont été sociabilisées dans des familles qui adhèrent à la fois à des valeurs traditionnelles et à des valeurs individualistes. Cette tension mérite d'être interrogée pour comprendre les parcours de ces femmes. Elles veulent suivre l'exemple de pères, parfois aussi de mères ou de sœurs, qui ont commencé à accéder à l'individuation : tels ces pères qui voyagent en Afrique, en Europe et qui inscrivent leurs filles à l'école publique; tel ce père du Maghreb qui confie sa boutique à sa fille adolescente, et qui la pousse ensuite à faire des études; tel ce père communiste qui discute de politique avec ses filles; telles ces mères qui commercent à travers l'Afrique et qui favorisent les 
sorties de leurs filles; telle cette mère qui invente des recettes culinaires alors que les autres femmes préparent tous les jours les mêmes plats (Veith, 2000); telle cette sœur haut fonctionnaire international qui incite son aînée à avoir un travail rémunéré. Le rôle des femmes de la famille est souvent plus discret parce qu'elles restent attachées à leur fonction de transmission des traditions ou qu'elles reportent sur leurs filles leurs aspirations à plus d'autonomie. Les femmes de l'enquête appartiennent à des générations charnières du point de vue des critères mis en exergue dans les récits, tels la scolarisation, l'âge au mariage, la possibilité de refuser le conjoint choisi par les parents. La place dans la fratrie est souvent essentielle : les cadettes réussissent là où les aînées ont échoué. Elles arrivent à transgresser les règles et les normes familiales et locales sans se mettre au ban de la société, à déroger aux manières de faire, d'être et de penser, socialement définies sans être pour autant sanctionnées. J'avance l'hypothèse que ces jeunes filles ont acquis lors de leurs socialisations initiales une disposition à jouer sur les tensions entre ces valeurs, à ruser, à tirer leur épingle du jeu pour atteindre leurs objectifs sans rompre avec leur famille.

Il s'agit de comprendre à partir de l'analyse de micro-situations comment une tendance globale, comme le développement de la scolarisation qui est l'une des clefs de l'individuation peut se diffuser en remettant en cause les normes admises localement. Cette transgression est alors le moteur d'un changement individuel et collectif. Elle devient aussi au niveau de ces femmes une disposition à ne pas se soumettre qu'elle transpose dans un autre contexte. Étayer ces hypothèses suppose alors d'analyser précisément des situations concrètes. Nous mettrons en perspective deux récits de vie, particulièrement explicites sur ces questions, et qui s'inscrivent avant la migration dans des contextes géographiques très éloignés : celui de Mina en Inde et celui de Binéta au Mali. Dans le récit de Mina, l'occurrence du « on », le «nous on » expriment l'appartenance à la ville, à la communauté, le respect de la coutume alors que l'usage du "je», du "c'est moi qui» affirme haut la volonté, la détermination à exister en tant qu'individu, quitte à transgresser la norme. Binéta, quant à elle, insiste explicitement dans son récit sur son désir d'affirmation de soi et sur son attachement à certaines valeurs holistes, comme la solidarité, acquises lors de sa socialisation initiale dans un contexte urbain où la communauté jouait un rôle important. Elle explique que, pendant son enfance dans son quartier, au cours des années cinquante et soixante, les personnes étaient nommées en tant que membre d'une famille, en tant que maillon d'une filiation. La façon d'appeler les personnes souligne un aspect de l'organisation sociale, ici holiste, dont elle est un indicateur. Elle révèle aussi l'une des facettes de l'identité personnelle pour et par autrui, en tension dans le récit biographique avec l'identité pour soi (Dubar, 2002). Cette nomination n'exclut pas pour autant d'autres formes d'identification, tout particulièrement dans une société locale en mutation qu'est cette capitale postcoloniale. La tension entre d'une part le respect des hiérarchies héritées et d'autre part l'accès à la modernité, caractérise les familles d'origine de ces deux femmes.

\section{Mina : une individuation en pointillé}

Mina est née en 1950 dans un comptoir français de l'Inde du Sud. Elle a vécu son enfance dans une ville côtière de marins et de pêcheurs qui n'envoyaient pas leurs enfants à l'école. Sa famille appartient à une caste élevée, celle des commerçants et des notables, qui dans la hiérarchie religieuse de l'Inde du Sud, est située juste après celles 
des Brahmanes. Le père de Mina est un petit entrepreneur en transport maritime et la famille sans être riche est à l'abri du besoin. Mina a été socialisée dans une société urbaine qui commence à être perméable à certaines valeurs individualistes, telle la réussite scolaire et économique, mais qui continue à respecter les hiérarchies de castes. Elle est la benjamine d'une famille de treize enfants. Les parents, les enfants célibataires, les fils mariés avec leur épouse et leurs enfants vivent tous ensemble dans une grande maison et Mina a la nostalgie de cette période heureuse où elle a été choyée par sa mère et ses belles-sœurs. Avec le développement de la scolarisation, le père a envoyé ses trois derniers enfants, deux garçons et Mina à l'école. Celle-ci est la seule à aimer aller à l'école où elle réussit brillamment.

\section{Une transgression exemplaire qui passe par des négociations individuelles}

- Tu vois, chez nous, lorsque la fille devient jeune fille, nous, on ne va pas à l'école. Être une jeune fille : c'est-à-dire avoir les premières règles.

Vous arrêtez l'école?

- Voilà quand les premières règles arrivent, ça veut que dire la fille devient jeune fille... Elle n'a pas le droit de sortir dehors, elle reste à la maison, tu vois. C'est pour ça qu'elles ont, voilà, arrêté l'école. Moi j'ai commencé à aller à l'école. Tout le monde est content, tu vois, dans ma famille, c'est moi qui allais à l'école. Mes frères sont, tous, fiers de moi. J'ai commencé à aller à l'école... je pensais : «Si je... une fois que je deviens jeune fille... » À l'âge de 11 ans... j'ai eu les premières règles. À ce moment-là, j'ai pleuré.

Ma mère a dit «pourquoi tu pleures?»

J'ai dit « Oui parce que je veux aller à l'école, je ne veux pas rester à la maison, je veux aller à l'école. »

Après mes frères, tous, ils ont dit: «Bon ça va. Normalement on n'a pas le droit d'envoyer, bon, si tu veux ». "Alors moi je suis partie comme ça. Après derrière moi, il y a des jeunes filles qui, comme ça, sont sorties aussi : aller à l'école, passer le bac, faire des études. Alors dans la cité, dans la ville, c'est moi la première femme qui...»

La vie de Mina bascule dans le monde des adultes à la mort de son père, tué dans une rixe où il s'était interposé. Ce décès qui provoque l'éclatement de la famille élargie coïncide avec la puberté de Mina, âgée de 11 ans. Avec la dispersion de la famille, la mère s'est retrouvée seule avec Mina, presque sans ressources. Elle envisagea même de se suicider en allant se noyer dans la mer avec Mina et y renonça au terme d'une longue marche sur la plage. Les deux plus jeunes fils, célibataires, les prirent alors en charge. Cette désagrégation familiale avait-elle finalement ouvert un espace de liberté pour cette adolescente de 11 ans déterminée à continuer sa scolarité ? Excepté la satisfaction des frères devant la réussite scolaire de leur petite sœur, aucun élément ne permettait d'avancer dans la compréhension de ce récit. Il fallait retourner poser la question à Mina. Celle-ci expliqua qu'en fait, son père était très fier d'elle et l'appelait «le professeur ». Elle ajouta alors un élément supplémentaire qui apporta une clef pour comprendre comment cela avait pu être possible. Sa famille n'était ni la plus riche, ni la plus influente. Cependant elle était crainte et respectée pour les réactions parfois belliqueuses des hommes du clan et personne dans la ville n'avait osé critiquer Mina. Paradoxalement dans ce cas, c'est une position dominante liée, entre autres, à la violence masculine - ici physique - qui va faciliter indirectement l'évolution de la condition féminine non seulement de la benjamine mais aussi d'autres jeunes filles de 
la ville qui se sont alors empressées de suivre cet exemple. Ce n'est pas tant la dislocation familiale qui a permis à Mina de déroger aux règles implicites de la coutume - l'enfermement des jeunes filles pour préserver l'honneur de la famille - mais la diffusion de la scolarisation et le fait d'appartenir à une famille respectée et crainte, sans minimiser toutefois la force de caractère de cette jeune fille à peine sortie de l'enfance et que ce deuil fait basculer dans le monde des adultes. Les circonstances et la position sociale de sa famille lui ont permis de se glisser dans la brèche ouverte par les évolutions en cours et d'infléchir l'orientation de sa vie personnelle. Elle a ainsi créé un précédent et elle est devenue un exemple à suivre. Forte de cette expérience, Mina poursuivit sa scolarité jusqu'au baccalauréat et occupa un poste d'institutrice pendant deux ans. Elle sut aussi à 18 ans déjouer habilement les arrangements matrimoniaux préparés par l'un de ses frères et imposer le conjoint de son choix, un homme de sa caste et de sa condition, en obtenant le soutien de sa mère et de sa grand-mère. Même si cette union respecte les règles de l'homogamie et donc de la reproduction sociale, elle marque une étape dans l'accès à l'individuation.

11 Dans cette évolution, la diffusion de la scolarisation et sa valorisation, dont témoigne la fierté du père et des frères devant la réussite de Mina, joue un rôle essentiel. Cependant Mina a pu enfreindre la règle de l'enfermement des jeunes filles pubères parce que, d'une part, sa famille commençait à concilier valeurs individualistes et valeurs holistes, d'autre part parce qu'elle était respectée et crainte. Dans la ville cette première transgression, introduit un changement individuel et collectif : individuel parce qu'il commence par l'action d'une personne et qu'il entre dans son patrimoine d'expériences réussies; collectif parce qu'il s'inscrit dans des transformations sociales globales et locales. La transgression se produit à un moment et dans un lieu où elle est socialement acceptable et acceptée ; elle met aussi en jeu les positions sociales ; enfin elle provoque un enchaînement de comportements similaires. Individuel et collectif sont inextricablement liés.

\section{Une individuation discontinue}

12 Peu après le mariage, le conjoint de Mina émigra vers la France où il espérait avoir un niveau de vie et de consommation supérieur et son épouse le rejoignit deux ans après en 1970. Mina qui ne parlait pas le Français et qui eut trois maternités rapprochées resta isolée pendant neuf ans dans une banlieue excentrée où les familles indiennes étaient peu nombreuses. Elle a fortement réduit en France sa natalité au vu des normes de sa famille restée en Inde puisqu'elle est la seule à avoir si peu d'enfants. Malgré les politiques autoritaires de maîtrise de la natalité en Inde, la réduction de celle-ci est très lente. L'attitude des membres de la famille de Mina, qui continuent à avoir de nombreux enfants, illustre cet écart entre la nouvelle règle imposée par l'État indien et les pratiques, entre la régle-contrainte et celle intériorisée par la famille, la norme que celle-ci continue à prescrire à ses membres. C'est en venant en France dans un pays qui encourage les naissances, que Mina réduit paradoxalement sa natalité au regard de celle de ses parents, de celle de ses frères et sœurs. En ayant trois enfants elle fait partie en France des familles nombreuses au sens de l'INSEE sans se percevoir comme telle. L'éloignement géographique ainsi que l'injonction de normes et règles contradictoires ouvre un espace de liberté pour décider de celle à laquelle se conformer. Le contrôle de la fécondité et le choix du nombre d'enfants qui posent la question de la maîtrise de son 
corps et de l'orientation de sa vie privée est l'une des facettes de l'individuation des femmes.

Cependant cette période correspond pour Mina à une phase de repli sur la sphère domestique, où elle dépend totalement de son conjoint. L'individuation n'est donc pas forcément un processus continu. Dans les sociétés individualistes, la sortie de la dépendance, en particulier à travers l'accès à l'instruction et à des moyens autonomes de subsistance, conditionne l'individuation. Pour les femmes, celle-ci se décline de façon spécifique puisque la dépendance s'inscrit dans des rapports de genre, dans des protections et des contrôles rapprochés dont les femmes cherchent à se dégager pour avoir plus d'emprise sur leur vie et établir des relations conjugales sur d'autres bases.

Le récit de Mina montre néanmoins sa capacité à rebondir après des périodes de replis en mobilisant non seulement ses ressources scolaires, sociales et culturelles mais aussi subjectives (Delcroix, 2001). Sur les conseils de l'institutrice de sa fille, Mina s'inscrit en 1979 à des cours de français où elle rencontre des voisines originaires de divers pays. Avec l'aide des formatrices françaises, elles décident de créer en 1980 une association féminine et multiculturelle dont elle est devenue l'un des piliers. Elles cherchent à résoudre ensemble les problèmes de leur quotidien: leur isolement linguistique et parfois social, l'illettrisme et parfois l'analphabétisme, les difficultés rencontrées dans les relations avec les institutions, dans le suivi de la scolarité des enfants, leur perte parfois d'indépendance financière suite à la migration. Elles cherchent aussi à avoir un lieu à elles dans la cité, à sortir de l'espace du logement pour retrouver une convivialité féminine. Les maris, et en particulier celui de Mina, ont une bonne image de l'association car ils savent que leurs épouses se retrouvent entre femmes. Mina y apprend à coudre et à habiller ses filles à moindre coût, à parler français, ce qui lui permet de faire seule, les courses, les démarches administratives, d'aller chez le médecin. Elle est donc mieux à même d'assumer son rôle traditionnel de mère de famille. Elle commence aussi à gagner de l'argent en gardant à l'association les jeunes enfants des femmes inscrites aux cours. Elle va suivre une formation d'animatrice. Avec d'autres femmes, africaines et maghrébines, elle monte treize ans plus tard un restaurant et elle accède alors progressivement à l'indépendance financière. Elles $\mathrm{y}$ professionnalisent des savoirs culinaires issus de leurs diverses cultures. Mina négocie avec son mari la possibilité de sortir du quartier dans la journée puis le soir pour aller travailler. Timide et réservée, elle n'hésite pas alors à prendre la parole en public, à la télévision pour défendre le dossier. Elle acquiert une capacité de parole et de mouvement. Paradoxalement alors qu'elle participe à des actions et à des projets collectifs dans une société « individualiste », elle reprend en France, une individuation qu'elle avait amorcée dans une société régie par le système des castes : une société dont l'analyse a permis à Louis Dumont de construire son modèle de l'homo hierarchicus et de l'holisme (1979).

Pour avoir porté à bout de bras pendant douze ans ce projet, pour s'être imposé, au nom de la survie du restaurant, des conditions de travail difficiles, des heures supplémentaires non rémunérées, des horaires flexibles, extensibles et décalés, Mina a dû s'arrêter pour raisons de santé. Après la fermeture du restaurant, suite à huit ans d'exercice, elle s'est repliée sur une fonction maternante en gardant ses petits-enfants afin de permettre à sa fille de continuer sa carrière professionnelle. Elle reporte alors sur cette dernière son aspiration à plus d'indépendance. Cependant parce qu'elle a toujours conservé des liens forts avec l'association, elle a pu y assumer une fonction 
d'interprète avec les familles tamoules arrivées récemment dans le quartier, un bénévolat qui devrait aboutir à la création d'un poste de médiatrice. Mina a décidé de rester en France avec ses filles et de ne pas suivre son mari lorsqu'il partira bientôt passer sa retraite en Inde.

L'histoire de Mina permet aussi d'avancer une autre hypothèse. Certaines périodes du cycle de vie des femmes sont moins propices à leur individuation et l'assignation à des fonctions maternantes auprès des jeunes enfants, et plus tard des petits-enfants, des parents âgés peut être un obstacle. L'insertion tardive de ces femmes sur le marché du travail, leurs trajectoires professionnelles courtes et discontinues, leurs faibles rémunérations ne garantissent pas leur indépendance financière au moment de la retraite. L'histoire de Mina montre à quel point l'individuation des femmes et en particulier celle des migrantes est un processus non linéaire, fragile et inachevé.

\section{Binéta : une individuation qui passe par l'accès à la citoyenneté}

Binéta est née en 1951 dans une colonie, le Soudan français. Elle vit à Bamako au sein d'une famille élargie qui regroupe environ 150 personnes autour de trois cours. Le père nourrit et loge ses quatre co-épouses et leurs vingt-six enfants, deux oncles polygames et leurs familles, des neveux orphelins, des personnes venues du village d'origine et de passage à la capitale. Binéta, née de la première épouse, tient le rôle de l'aînée des filles : sa sœur, née d'un premier mariage de la mère et de neuf ans plus âgée, a quitté la famille après avoir été mariée à un cousin. Dans le récit, l'influence du père est centrale, laissant en arrière-plan le rôle plus discret des femmes.

Le père de Binéta est un riche commerçant qui a fait fortune après la Seconde Guerre mondiale en important au Mali des produits étrangers achetés lors de voyages en Europe. Sa prospérité lui a permis de faire venir à la capitale ses deux frères et leurs familles, de leur donner une place dans la gestion de ses affaires : l'aîné s'occupait d'une boutique et des terres cultivées pour nourrir la famille; le benjamin d'une filiale de transports. Ce père, originaire de la campagne et qui n'avait pas été scolarisé, a toujours su prendre des initiatives et faire preuve d'un grand dynamisme. Convaincu de l'importance de l'enseignement, il avait inscrit ses enfants, garçons et filles, à l'école publique française.

\section{Le contournement de la norme revendiquée collectivement}

« Moi j'ai eu la chance d'aller à l'école parce que dans mon temps, parce que je suis des années cinquante, les filles n'allaient pas à l'école. C'étaient les garçons qui allaient à l'école. Pourquoi ? Parce que les parents trouvaient que ce n'était pas nécessaire d'envoyer les filles parce qu'elles sont destinées à être ménagères à la maison. Et par contre ça n'a pas été le cas pour nous. Nous, on a commencé à aller à l'école, on a fait d'abord une première année à l'école française et ensuite... le frère le plus grand de mon père a fait comprendre que, nous, on n'en a pas besoin : les filles n'ont pas besoin d'aller à l'école. Par contre on peut nous mettre à l'école coranique, arabe, pour apprendre la religion, pour qu'on puisse plus tard dans notre famille transmettre la religion... tout ce qu'une femme doit transmettre. Donc on a quitté l'école française et puis on a fait l'école arabe pendant cinq ans. [...] Après... on a protesté comme quoi on a droit, aussi, d'aller à l'école française. » 
19 Cinq ans plus tard, Binéta, les sœurs et les cousines de son âge ont contesté collectivement la décision de l'oncle aîné : elles ont convaincu le père qui ne demandait qu'à l'être et qui finalement osa désobéir à son frère, contrairement à la coutume.

«Peut-être que ça allait dans le même sens que celui que mon père voulait. Ce qui est paradoxal, bon mon père, c'est un commerçant, c'est un homme qui a beaucoup voyagé, il est venu ici à la fin de la guerre parce qu'il... allait acheter des marchandises pour vendre au pays. Il a fait pas mal de pays d'Europe. Donc il a compris que les filles, aussi, ont droit de savoir autant que les garçons. Donc il nous a retourné à l'école, à l'école française. »

20 Ce père finança même l'inscription des plus grandes dans le privé afin qu'elles puissent rattraper les cinq années de retard et il incita Binéta à poursuivre sa scolarité jusqu'au baccalauréat: il voulait qu'elle se forme afin qu'elle puisse travailler à la douane et ainsi faciliter ses activités d'import. Binéta s'inscrivit en fait dans une école de la poste pour devenir agent d'exploitation.

21 Ce père vit un dilemme : d'une part il est ouvert à la modernité qu'il souhaite pour ses enfants et d'autre part il ne cherche pas à remettre en cause le fonctionnement collectif et traditionnel de la famille, fondé sur le maintien des hiérarchies liées, entre autres, à la place dans la fratrie. C'est malgré lui qu'il est amené à le faire, ses convictions personnelles l'emportant finalement sur le respect de la tradition. Ses voyages ont favorisé son ouverture à d'autres systèmes de valeurs tout en permettant son enrichissement et sa mobilité sociale ascendante. Cependant celle-ci dans cet environnement est alors devenue un obstacle aux prémices d'une individuation des femmes. Un négociant aussi fortuné devait montrer qu'il était capable de nourrir sa nombreuse famille, d'aider sa parentèle. C'est là un signe de prestige social. Les épouses ont alors dû renoncer à leurs petits commerces sur le marché. Le père de Binéta refusa aussi que ses filles et nièces se fassent de l'argent de poche en vendant des fruits et des brochettes dans la rue. Puis il se laissa convaincre et finalement les épaula dans leurs initiatives et dans leur volonté d'être plus autonomes.

La solidarité communautaire et les protections qu'elle apporte est l'un des fondements du modèle des sociétés traditionnelles puisqu'elle s'exerce entre membres du groupe en échange d'un respect de l'ordre établi. Ici, dans ce contexte de montée de l'individualisme, elle renforce paradoxalement le pouvoir économique de ce cadet qui fait vivre toute la famille et le conduit à supplanter le pouvoir hiérarchique de l'aîné. Dans un second temps avec l'élargissement de la famille et le développement de la scolarisation, le père n'est plus en mesure d'assumer cette solidarité, de nourrir tous ses parents et de financer les études des enfants. Il est alors obligé d'accepter que ses épouses aient à nouveau des activités commerciales et participent à l'entretien de la famille. On voit alors émerger le rôle économique des mères qui voyagent à travers l'Afrique.

23 Celles-ci facilitent aussi les sorties des filles dans une famille réputée pour son « orthodoxie ». Ce père, ouvert à la modernité, tient néanmoins, le rôle hérité du système patriarcal : il incarne l'autorité et la domination masculine (Bourdieu, 1998) ; il est le garant de l'honneur familial en particulier de celui de ses épouses et de ses filles, ce qui suppose de donner à ces dernières une éducation stricte, de contrôler leurs déplacements. C'est pourquoi les filles sortent en cachette, pour faire du théâtre, jouer au basket, aller à des thés dansants l'après-midi. Elles le font alors avec la complicité 
des tantes et des mères. Celles-ci plus souples, veulent laisser à leurs filles un peu de cette liberté dont elles n'ont pu jouir.

\section{Le choix du conjoint}

24 C'est à l'occasion d'une soirée que Binéta a rencontré son mari, un parent éloigné venu faire des études à la capitale. Binéta est la première de la famille à pouvoir choisir son conjoint : sa sœur aînée, de 9 ans plus âgée, et une cadette, mariée plus jeune avaient dû accepter des unions arrangées par la famille: le père n'avait pas alors osé s'y opposer. "J'ai décidé de choisir pour moi » dit Binéta, juste après avoir rappelé qu'elle avait fait des études. Elle refusa plusieurs prétendants et en particulier celui, choisi par l'oncle benjamin, qui souhaitait renforcer l'alliance qu'il avait contractée avec la famille de sa nouvelle épouse. Binéta explique alors comment elle a obtenu le soutien d'une tante aînée et influente, à travers l'intervention d'une cousine. Celle-ci a plaidé la cause de Binéta en insistant sur la détermination de celle-ci : la famille en s'obstinant risquait d'être responsable devant Dieu si Binéta en persistant dans son refus «se rebellait et tournait mal ». Le père, cette fois, donna raison à Binéta et son exemple fut suivi par les autres filles. Comme pour Mina, ce mariage qui respecte les règles de l'endogamie, marque néanmoins une étape dans l'accès à l'individuation.

\section{La formation politique}

La mobilisation politique est un dernier élément à prendre en compte. Dans son récit Binéta évoque l'encadrement politique de la jeunesse malienne sous le régime socialiste de Modibo Keita, premier président du Mali de 1960 à 1968.

«Je faisais partie des pionniers. Ça s'appelait à l'époque, "les pionniers de l'école". Enfant je trouvais ça très intéressant mais en fait c'était du bourrage de crâne. Moi, j'étais gamine, j'étais emballée parce qu'on faisait plein de choses. On ne savait pas pourquoi on les faisait (rire de Binéta). Par exemple on lançait des slogans contre les impérialistes, des trucs comme ça. Mais il y avait aussi les activités sportives et culturelles, le théâtre, l'éducation civique populaire, qui étaient destinées à la population par rapport à la propreté, par rapport au civisme, par rapport à plein de choses. »

Binéta insiste sur l'endoctrinement politique et sur la dérive totalitaire du gouvernement. En même temps elle montre comment, au début du régime, la mobilisation de la population et en particulier celle de la jeunesse, sur des projets collectifs d'intérêt commun a contribué à forger son goût pour les affaires publiques. Elle a aussi appris le sens de la critique en prenant du recul par rapport à l'action économique du gouvernement et surtout par rapport à la répression des milices populaires. Après le coup d'État militaire qui renversa le pouvoir socialiste en 1968 et instaura une nouvelle dictature, elle participa à la révolte des jeunes qui contestaient l'instauration d'un parti unique : «On n'avait pas le droit de s'exprimer, de s'affirmer. » Elle a alors appris à investir l'espace public en manifestant pour revendiquer le droit d'expression. 


\section{Une trajectoire professionnelle précarisée}

En 1974 Binéta décida de rejoindre son mari venu en France pour suivre des études. Comme pour Mina, l'adaptation est difficile et Binéta souffre au début de la solitude. Sur les conseils de son mari, elle cherche rapidement un travail ; elle suit des cours du soir et passe un CAP de comptabilité en vue du retour au pays. Elle enchaîne les périodes de chômage et les emplois précaires, non qualifiés et peu rémunérés : des emplois de femmes de ménage, de manutentionnaire, d'aide ménagère, d'aide cuisinière. Elle perd les deux seuls emplois qui se soient stabilisés, des emplois d'aide ménagère auprès de personnes âgées qui lui apportaient pourtant des satisfactions relationnelles : le premier à la suite de sa première grossesse en 1977 ; le second en 1989, après sept ans d'exercice, en raison d'une incapacité médicale.

Lorsque son mari décide en 1989 de retourner au Mali, Binéta préfère rester en France avec leurs trois enfants afin de leur assurer de meilleures conditions de vie. Sa priorité est de gagner suffisamment d'argent. Elle est désormais seule pour faire vivre la famille : un salaire de fonctionnaire malien ne permet pas à son mari de lui envoyer de l'argent en France. Elle envisage de s'orienter vers le secteur médical, passer des concours pour avoir un emploi stable, quitte à se contenter d'une fonction routinière. Cependant depuis le départ de son mari, Binéta veut combler un vide. Elle aspire à avoir des contacts humains en ayant un travail à caractère social, au service des immigrés qu'elle voit si désemparés et démunis dans les administrations et dont elle reste solidaire même si elle a acquis la nationalité française. Elle s'investit dans les associations locales de locataires, de parents d'élèves et dans une association multiculturelle où les femmes se sont fortement impliquées. Elle suit alors une formation pour pouvoir y donner des cours d'alphabétisation. En développant cette nouvelle activité, elle crée son emploi. Elle privilégie alors le contenu valorisant de son travail par rapport à la rémunération qui reste faible.

Individuation et mobilisation politique et associative sont ici étroitement liées. Dans son récit, Binéta associe la formation de l'esprit critique et son sens de l'engagement politique à son désir d'autonomie qu'elle a pu développer au sein de sa famille d'origine. L'accès à la citoyenneté est en effet l'une des dimensions de l'individuation : il peut être à la fois l'aboutissement et l'un des moyens du processus. Parce qu'elle a commencé à accéder au savoir, à l'indépendance financière, à plus de liberté de mouvements, en remettant en cause les normes sociales, comme le rôle traditionnel des femmes, elle a aiguisé son sens critique. Parce qu'elle s'est battue avec d'autres pour y arriver, elle consolide son sens de la mobilisation collective. Sa conscience de l'intérêt commun trouve aussi son origine dans l'importance accordée à la communauté dans les sociétés traditionnelles. Celle-ci peut alors être redéfinie dans une capacité à se mobiliser au sein d'un sous-groupe, ici les filles scolarisables, et à contester les normes établies et les valeurs qui légitiment les hiérarchies sociales et sexuées héritées. Avec la transformation du contexte, des valeurs qui sont au fondement de l'holisme sont redéfinies dans une conception plus individualiste, favorisant alors l'individuation. En France, accorder de l'importance à la solidarité, qui ne s'exerce plus en contrepartie d'une acceptation des dominations, est l'un des moteurs de l'individuation de Binéta.

Ici individuation et engagement collectif ne sont pas contradictoires puisque l'apprentissage de la démocratie suppose de se penser comme un individu autonome, capable d'avoir un avis différent. L'individuation a plutôt été développée dans la vie 
privée et a permis le développement de l'esprit critique dans la mobilisation politique qui devient alors une force potentielle de contestation y compris au sein de la famille d'origine.

31 On comprend comment en France Binéta a pu militer avec son mari dans les mouvements étudiants africains, s'intéresser au parti communiste français avant de s'en détourner après l'exclusion de membres du Comité central et la destruction d'un foyer immigré. Elle s'est alors investie dans les associations locales et dans les mouvements sociaux. En 2001, elle accepte de figurer sur une liste électorale associative et elle est élue conseillère municipale.

\section{Conclusion}

La comparaison des récits de Mina et de Binéta met en lumière la domination masculine qui traverse des contextes géographiques, sociaux et religieux très éloignés. Les récits montrent aussi l'imbrication des valeurs holistes et individualistes dans des sociétés urbaines et coloniales ou post-coloniales.

Dans les récits, l'analyse des situations d'interaction, où se nouent les relations sociales inscrites dans l'ordre structurant des rapports sociaux (de Rudder, Poiret et Vourc'h, 2000), permet de comprendre comment les tensions entre valeurs holistes et valeurs individualistes ouvrent des interstices où ces femmes ont pu se glisser pour infléchir le cours de leur vie. Au-delà des ressources sociales culturelles mobilisées, c'est en effet une capacité à conserver des liens forts avec la "communauté » d'origine tout en la maintenant à distance, une capacité à jouer sur ces tensions entre systèmes normatifs contradictoires, voire à les articuler, qui ouvrent des perspectives pour penser des cheminements jonchés d'obstacles que vivent ces femmes en France: déclassement social, relégation dans des quartiers défavorisés, stigmatisation, discriminations et précarisation sociale. Des associations locales, féminines et multiculturelles, sont en France les contextes qui favorisent la mobilisation de ressources sociales et culturelles et de dispositions acquises pendant les socialisations antérieures. L'engagement dans ces collectifs électifs est le ressort de l'action de ces femmes. Il leur permet de construire des projets et de tenter d'accéder à plus d'individuation même si celle des femmes et en particulier celles des migrantes se révèle être un processus inachevé, fragile et menacé par la précarisation sociale. Dans des quartiers populaires, il est une voie spécifique d'individuation qui joue la carte de la citoyenneté.

\section{BIBLIOGRAPHIE}

APPAY Béatrice et LÉOMANT Christian Dirs. (1999) Précarisation salariale et précarisation familiale, Rapport de fin de contrat CNAF/Béatrice Appay, Christian Léomant, Nicole Sotteau-Léomant, Blandine Veith, Paris, Iresco, $284 \mathrm{p}$. 
APPAY Béatrice et THÉBAUD-MONY Annie Dirs. (1997) Précarisation sociale, travail et santé, Paris, Iresco, $575 \mathrm{p}$.

BERTAUX Daniel (1999) Les récits de vie, Paris, Nathan, 127 p.

BIRNBAUM Pierre et LECA Jean Dirs. (1986) Sur l'individualism : théories et méthodes, Paris, Presses de la FNSP, 379 p.

BOURDIEU Pierre (1998) La domination masculine, Paris, Seuil, 139 p.

CASTEL Robert (2001) Propriété privée, propriété sociale, propriété de soi : entretien sur la construction de l'individu moderne, Paris, Fayard, 210 p.

DELCROIX Catherine (2001) La famille Nours, Paris, Payot, 253 p.

DELCROIX Catherine et BERTAUX Daniel (2000) La dernière vague : le capital biographique d'une génération d'immigrés du Maghreb, Écarts d'identité, 92.

DUBAR Claude (2002) L'articulation des temporalités dans la construction des identités personnelles, Temporalistes, 44, pp. 42-49

DUMONT Louis (1979) Homo hierarchicus : le système des castes et ses applications, Paris, Gallimard, $490 \mathrm{p}$.

DUMONT Louis (1991) Essais sur l'individualisme : une perspective anthropologique sur l'idéologie moderne. Paris, Seuil, $310 \mathrm{p}$.

EHRENBERG Alain (1998) La fatigue d'être soi : dépression et société, Paris, O. Jacob, 318 p.

ELIAS Norbert (1997) La société des individus, Paris, Fayard, 301 p.

KAUFMANN Jean-Claude (2001) L'entretien compréhensif, Paris, Nathan, 127 p.

LAHIRE Bernard (1998) L'homme pluriel : les ressorts de l'action, Nathan, $271 \mathrm{p}$.

LIPIANSKY Edmond Marc, TABOADA-LEONETTI Isabelle et VASQUEZ Ana (1990) Introduction à la problématique de l'identité in Stratégies identitaires, Paris, l'Harmattan, pp. 7-26.

MARSHALL Thomas Humphrey (1950) Citizenship and Social Class, in Citizenship and Social Class and Other Essays, Cambridge, (UK), Cambridge University Press, pp. 1-85.

RUDDER Véronique de, POIRET Christian et VOURC'H François (2000) L'inégalité raciste : l'universalité à l'épreuve de la République, Paris, PUF, 213 p.

SINGLY François de (2000) Libres ensemble : l'individualisme dans la vie commune, $253 \mathrm{p}$.

SINGLY François de (2001) «La naissance de l'individu individualisé et ses effets sur la vie conjugale et familiale » in SINGLY François de Dir., Famille et individualisation, l'Harmattan, pp. 5-14.

VEITH Blandine (1998) Femmes immigrées : de l'implication associative à la dynamique de travail, Cahiers du Gedisst, 23, pp. 65-85.

VEITH Blandine (1999) Précarisation et association, in Précarisation salariale et précarisation familiale, rapport de fin de contrat CNAF/Béatrice Appay, Christian Léomant, Nicole SotteauLéomant, Blandine Veith, Paris, Iresco, pp. 201-263.

VEITH Blandine (2000) Femmes migrantes et pratiques culinaires associatives, Bastidiana, 31-32, décembre. 


\section{RÉSUMÉS}

L'auteur traite de la construction de l'individu moderne à travers l'engagement associatif de femmes migrantes précarisées. La perspective de genre prend en compte les effets des migrations internationales de sociétés post-coloniales vers la France. Ces migrations sont à la fois des freins et des incitations, voire des injonctions, à maîtriser le cours de sa vie alors que les conditions objectives ne sont pas remplies pour réaliser ces aspirations. L'article analyse deux récits de vie, ceux de deux femmes engagées en France dans des associations locales et multiculturelles. La première est originaire d'un ancien comptoir de l'Inde ; la seconde, du Mali. Pour poursuivre une individuation commencée avant la migration, ces femmes cherchent à concilier aspiration à plus d'autonomie et maintien de liens avec leurs "communautés». Elles mobilisent des ressources subjectives qu'elles ont développées lors de leur socialisation initiale en se glissant dans les interstices qu'ouvrent les tensions entre normes holistes et individualistes.

\section{Migrant Women's Involvement and Individuation in Associations.}

The author deals with the construction of modern individual through the involvement in associations of migrants women in precarious conditions. The gender wiewpoint takes into account the effects of migration from postcolonial societies to France. The article analyses the life stories of two women involved in local and multicultural associations. The first woman comes from an old Indian trading post, the second comes from Mali. To continue an individuation begun before migration, these women try to reconcile theire desir to be more self-sufficient and the maintening of links with their communities. They use subjective resources, they have developed during their initial socialisation by exploiting openings offered by the the tensions between holistic and indvidualistic norms.

Compromiso asociativo e individuación de mujeres inmigrantes.

El autor trata de la construcción del individuo moderno a través del compromiso asociativo de mujeres inmigrantes en situación precaria. La perspectiva de género toma en cuenta los efectos de las migraciones internacionales de sociedades post-coloniales en dirección de Francia. Estas migraciones constituyen al mismo tiempo frenos e incitaciones, e incluso in-junciones, al control del curso de la propia vida incluso en el caso de que las condiciones objetivas no estén reunidas. El artículo analiza los relatos de vida de dos mujeres comprometidas en Francia con asociaciones locales y multiculturales. La primera es originaria de un antiguo puesto comercial de la India ; la segunda, de Malí. Para proseguir con una individuación cuyo comienzo data de antes de la migración, estas mujeres intentan conciliar su aspiración a más autonomía con la conservación de los lazos con sus propias comunidades. Ambas mujeres utilizan recursos subjetivos que construyeron durante su socialización inicial colándose así en los intersticios que abren las tensiones entre normas holistas e individualistas.

\section{INDEX}

Mots-clés : associations, femmes, histoire de vie, Indiens, Maliens, précarité Index géographique : France

\section{AUTEUR}

\section{BLANDINE VEITH}

Ingénieur de Recherche, Sociologue CNRS, Ladyss, Université de Paris 10 Bât K - 200, av. de la République, 92001 Nanterre Cedex, bveith@u-paris10.fr 